\title{
Introduction to the focused section on new trends of autonomous robot navigation
}

\author{
Xuebo Zhang ${ }^{1}$ \\ Published online: 1 June 2021 \\ (c) The Author(s), under exclusive licence to Springer Nature Singapore Pte Ltd. 2021
}

Autonomous navigation is a fundamental task in the field of intelligent robotics, playing a vital role in various emerging applications such as automated warehouses, autonomous exploration of hazard environments, unmanned driving, and so on. A rapid development of artificial intelligence (AI) in recent years leads to the evolution of traditional perception, planning and control approaches more into data-based techniques with learning and adaptation capabilities. These technologies are potentially effective in highly dynamic and unstructured complex environments from indoor places to outdoor environments. Thus, incorporating these advanced methods into autonomous robot navigation will increase the autonomy and intelligence level of robotic systems. This Focused Section is dedicated to new advances in both theoretical approaches and practical applications of perception, planning and control for autonomous mechatronic and robotic systems.

The Focused Section consists of 12 research papers covering sensing and detection algorithms of autonomous robotic systems, intelligent motion planning methods, novel robotic control schemes and promising applications of AIbased robotic systems.

The first part of the Focused Section investigates intelligent sensing and detection algorithms of autonomous robotic systems. Zhang et al. propose a novel hierarchical and efficient network (HENet) to re-identify persons to faciliate the mobile robot navigation. The approach has better efficiency and robustness compared with existing identification methods. Xie et al. develop a loosely-coupled lidar-inertial odometry and mapping method for real-time state estimation of the mobile robot. Lin et al. design a monitoring scheme of materials in the warehouse based on distributed system and edge computing. The scheme can calculate the volume of materials in the warehouse and monitor whether materials

Xuebo Zhang

zhangxuebo@nankai.edu.cn

1 College of Artificial Intelligence, Nankai University, Tianjin, China are lacked in real time. Shen et al. present a new lane line detection and recognition approach for unmanned vehicles based on dynamic region of interest (ROI) and a modified firefly algorithm. This approach has satisfactory detection accuracy in the challenging night and rainy conditions.

The second part of the Focused Section presents some novel motion planning methods for unmanned vehicles and robotic arms. Zhu et al. propose a flexible cooperative navigation $(\mathrm{CN})$ scheme for the multiple unmanned ground vehicles system, in which a novel $\epsilon$-constraint probability collectives (PC) method is introduced to balance the high-quality cooperative optimization and acceptable computational speed, while guaranteeing the safety of the $\mathrm{CN}$. Zhang et al. develop a decentralized motion planning approach for multi-quadrotor autonomous navigation, wherein the inter-collision-free is achieved by formulating the collision risk as a penalty term of the cost function. Li et al. propose a hybrid obstacle avoidance method for robotic arms by combining the reinforcement learning (RL) with artificial potential field method (APFM). Compared with the traditional APFM, this method can be used to avoid dynamic obstacles and obstacles close to the target.

The third part of the Focused Section shows two cases devoted to robotic control methods. Li et al. comprehensively review the latest visual servoing methods of mobile robots and summarize several constructive guidelines for future research, in which learning-based and multi-moduleintegration-based visual servoing schemes are regarded as promising research trends. $\mathrm{Li}$ et al. design a new nonsingular terminal sliding mode control (NTSMC) scheme for trajectory tracking control of omnidirectional mobile manipulators.

The last part of the Focused Section introduces several interesting and promising applications of AI-based robotic systems. Abed et al. propose an innovative deep learning framework for automated bean leaves disease detection. This framework can detect infected leaves from input bean images and diagnose the specific diseases within the detected leaves, which has a wide application prospect in modern agriculture. 
Yang et al. design a low-cost, real-time and robust wind measurement system by identifying the airflow rope's fluttering based on computer vision (CV). This $\mathrm{CV}$-wind-sensor can be simply deployed on unmanned sailboats to measure the wind speed and direction. Li et al. introduce a new indoor way-finding method for visually impaired people (VIP) by fusing the measurement information of IMU and the magnetic tensor sensor. This method can guide the VIPs efficiently and accurately to destinations in different indoor scenarios.

The 12 papers, carefully selected from 37 received submissions, illustrate recently developed algorithms of perception, planning and control for robotic systems. Most of the proposed solutions integrate AI-based techniques (such as deep learning and reinforcement learning) with traditional approaches, reflecting the new trends observed today in the autonomous robot navigation field and leading to promising results.

We wish to take this opportunity to thank all of the authors and all anonymous reviewers for their valuable time and efforts spent to make this focused section possible. In particular, we would like to express our sincere gratitude to following guest editors (in alphabetical order) for their hard and careful work during the peer review process and proofreading of the editorial.

\section{Guest Editors}

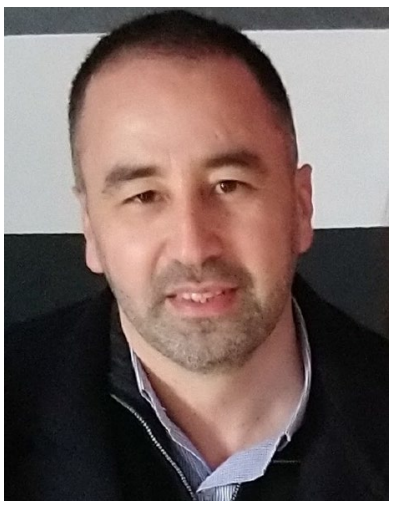

Lounis Adouane is a full Professor at Université de Technologie de Compiègne. He received the $\mathrm{Ph} . \mathrm{D}$. degree in automatic control from the FEMTO-ST Laboratory, ENSMM, in 2005. During his Ph.D. studies, he deeply investigated the field of mobile multirobot systems; especially those related to bottom-up and decentralized control architectures. In 2005, he joined the Ampere Laboratory, INSA Lyon, and he studied hybrid (continuous/ discrete) control architectures applied to cooperative mobile robots arms. In 2015, he obtained an HDR (habilitation to steer research in robotics) from Blaise Pascal University. From 2006 to 2019, he was an Associate Professor at the Institut Pascal, Polytech Clermont-Ferrand. Currently, Lounis Adouane hold a full Professor position at Heudiasyc, UMR 7253 CNRS / UTC. Between 2018 and 2019, he was the head of ISPR Group (Images, Perception Systems and Robotics, 80 members) at Institut Pascal. Since 2018, Prof. Lounis Adouane is a member of the Technical Committee of IFAC Intelligent Autonomous Vehicles and serves in the Editorial Board of the Journal of Intelligent and Robotic Systems. Prof. Lounis Adouane is the author/coauthor of more than 120 refereed international papers and two books. His main research interest include planning and control of mobile robots / intelligent vehicles (autonomous and clean), decision-making under uncertainty, hybrid (continuous/discrete) and hybrid (reactive/cognitive) multi-controller architectures, Lyapunov based synthesis and stability, cooperative multi-robot systems, artificial intelligence (e.g., MDP or MAS approaches), energy management (e.g., with optimal control or neuro-fuzzy approaches). (E-mail: Lounis.Adouane@utc.fr)

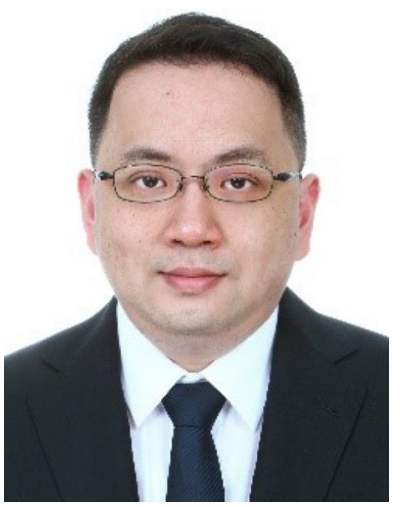

Chun-Yeon Lin received the M.S. degree in mechanical engineering from Stanford University in 2011; and the Ph.D. degree in mechanical engineering from George W. Woodruff School of Mechanical Engineering, Georgia Institute of Technology in 2017. Currently, he is an assistant professor in the Department of Mechanical Engineering, National Taiwan University. His current research interests include mechatronics, electromagnetic system and modeling. (E-mail: chunyeonlin@

ntu.edu.tw)

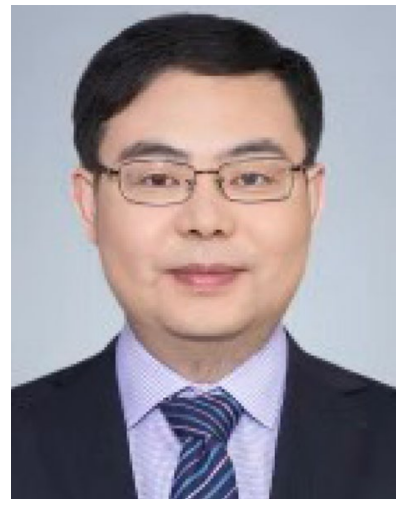

Yong Liu is Professor of Institute of Cyber-Systems and Control, Zhejiang University, Director of Research Center for Intelligent Driving and Future Transport, College of Control Science and Engineering, Zhejiang University. He was selected as the national young top-notch talent of "Ten Thousand Talents Program" in 2019, the outstanding young scientific and technological talents of Zhejiang Province and the 151 talent project of Zhejiang Province. His research interests include autonomous robots and intelligent systems, robot perception systems, autonomous robot planning and navigation control, visual recognition and pattern recognition, SLAM technology and multi-sensor fusion technology. (E-mail: yongliu@iipc.zju.edu.cn)

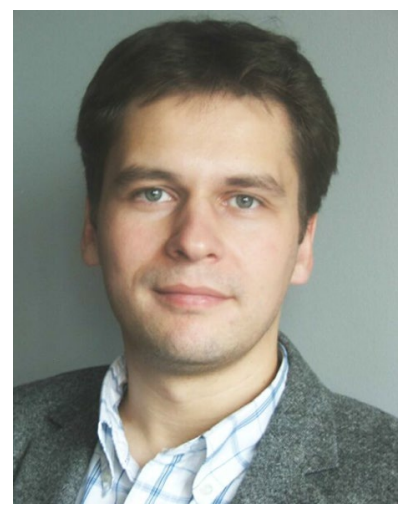

Maciej M. Michalek is currently an Associate/Ac-ademic Professor of PUT in the Institute of Automatic Control and Robotics, where he serves as a deputy director for scientific affairs. His current research interests concern modeling, control design, and control applications in the area of nonholonomic systems, mobile robots, articulated vehicles, $\mathrm{N}$-trailer structures, and intelligent/automated vehicles. $\mathrm{He}$ is a Senior Member of IEEE and the Chair of the Polish Chapter of IEEE Robotics \& Automation Society (in years 2020-2022). Currently he is a member of the Conference Editorial Board for the IEEE Control Systems Society, an Executive Board Member of the Journal of Intelligent \& Robotic Systems, and an Associate Editor for the Journal of the Franklin Institute. (E-mail: maciej.michalek@put. poznan.pl) 


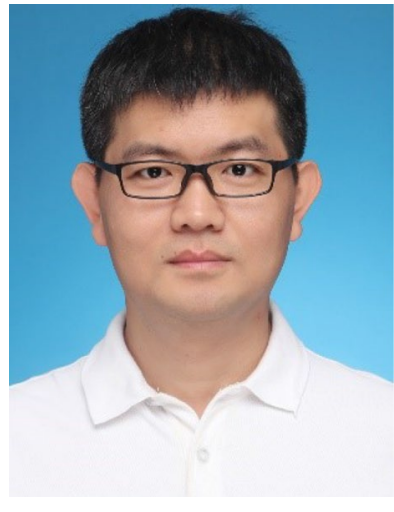

Bailing Tian is currently a professor at the School of Electrical and Information Engineering, Tianjin University. He was an academic visitor at the School of Electrical and Electronic Engineering, University of Manchester from June 2014 to June 2015. His main research interests include intelligent decisionmaking for multi-UAVs, motion planning of autono-mous robotics, finite time control and its application in UAV. (E-mail: bailing_tian@tju.edu.cn)

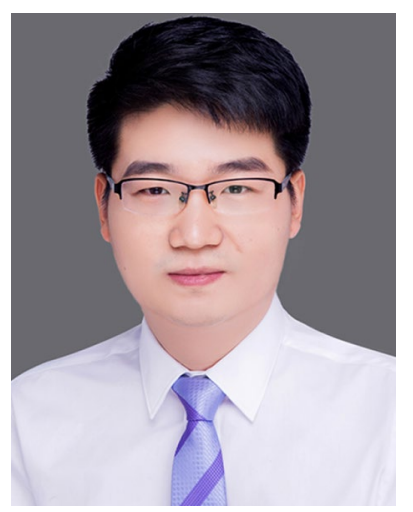

Xuebo Zhang received the B.Eng. degree in automation from Tianjin University, Tianjin, China, in 2006, and the Ph.D. degree in control theory and control engineering from Nankai University, Tianjin, China, in 2011. From 2014 to 2015, he was a Visiting Scholar with the Department of Electrical and Computer Engineering, University of Windsor, Windsor, ON, Canada. He was a Visiting Scholar with the Department of Mechanical and Biomedical Engineering, City University of Hong Kong, Hong Kong, in 2017. He is currently a Professor with the Institute of Robotics and Automatic Information System, Nankai University, and Tianjin Key Laboratory of Intelligent Robotics, Nankai University.
His research interests include planning and control of autonomous robotics and mechatronic system with focus on time-optimal planning and visual servo control; intelligent perception including robot vision, visual sensor networks, simultaneous localization and mapping (SLAM); reinforcement learning and game theory. Dr. Zhang is a Technical Editor of IEEE/ASME Transactions on Mechatronics and an Associate Editor of ASME Journal of Dynamic Systems, Measurement, and Control.

Publisher's Note Springer Nature remains neutral with regard to jurisdictional claims in published maps and institutional affiliations. 